\title{
FORWARD-BACKWARD STOCHASTIC DIFFERENTIAL EQUATIONS AND PDE WITH GRADIENT DEPENDENT SECOND ORDER COEFFICIENTS
}

\author{
ROMAIN ABRAHAM - OLIVIER RIVIERE
}

\begin{abstract}
We consider a system of fully coupled forward-backward stochastic differential equations. First we generalize the results of Pardoux-Tang [6] concerning the regularity of the solutions with respect to initial conditions. Then, we prove that in some particular cases this system leads to a probabilistic representation of solutions of a second-order PDE whose second order coefficients depend on the gradient of the solution. We then give some examples in dimension 1 and dimension 2 for which the assumptions are easy to check.
\end{abstract}

AMS Classification numbers: 60H10, 60H 30

Keywords: forward-backward stochastic differential equations, partial differential equations.

\section{INTRODUCTION}

Let us consider the following Partial Differential Equation:

$$
\begin{cases}\frac{\partial u}{\partial t}(t, x)+L u(t, x)+g(t, x, u(t, x),(\nabla u \sigma)(t, x))=0 & \forall(t, x) \in[0, T) \times \mathbb{R}^{d}, \\ u(T, x)=h(x) & \forall x \in \mathbb{R}^{d},\end{cases}
$$

where $u:[0, T] \times \mathbb{R}^{d} \rightarrow \mathbb{R}^{p}, h: \mathbb{R}^{d} \rightarrow \mathbb{R}^{p}, g:[0, T] \times \mathbb{R}^{d} \times \mathbb{R}^{p} \times \mathbb{R}^{p \times d} \rightarrow \mathbb{R}^{p}$, and $L u(t, x)=\frac{1}{2} \sum_{i, j}\left(\sigma \sigma^{*}\right)_{i j}(t, x, u) \frac{\partial^{2} u}{\partial x_{i} \partial x_{j}}+\sum_{i} f_{i}(t, x, u, \nabla u \sigma) \frac{\partial u}{\partial x_{i}}$

where $\sigma:[0,+\infty) \times \mathbb{R}^{d} \times \mathbb{R}^{p} \rightarrow \mathbb{R}^{d \times d}$ and $f:[0,+\infty) \times \mathbb{R}^{d} \times \mathbb{R}^{p} \times \mathbb{R}^{p \times d} \rightarrow \mathbb{R}^{d}$.

We know that we can represent (with some assumptions) the solutions of (1) with the solution of a Forward Backward Stochastic Differential Equation (FBSDE in short). Let $\left\{B_{t}, t \geq 0\right\}$ be a Brownian motion in $\mathbb{R}^{d}$ and let us consider the FBSDE:

$$
\left\{\begin{array}{l}
X_{s}^{t, x}=x+\int_{t}^{s} f\left(r, X_{r}^{t, x}, Y_{r}^{t, x}, Z_{r}^{t, x}\right) d r+\int_{t}^{s} \sigma\left(r, X_{r}^{t, x}, Y_{r}^{t, x}\right) d B_{r} \\
Y_{s}^{t, x}=h\left(X_{T}^{t, x}\right)+\int_{s}^{T} g\left(r, X_{r}^{t, x}, Y_{r}^{t, x}, Z_{r}^{t, x}\right) d r-\int_{s}^{T} Z_{r}^{t, x} d B_{r}
\end{array}\right.
$$

where $X^{t, x}:[t, T] \rightarrow \mathbb{R}^{d}, Y^{t, x}:[t, T] \rightarrow \mathbb{R}^{p}$ and $Z^{t, x}:[t, T] \rightarrow \mathbb{R}^{p \times d}$ are adapted to the filtration generated by $\left(B_{s}-B_{t}, s \geq t\right)$.

Then the deterministic function $u(t, x)=Y_{t}^{t, x}$ is a viscosity solution of (1). This has led to a huge amount of work. We refer to Pardoux-Tang [6], Ma-Yong [4] and the references therein for more precise results on the subject.

The goal of this paper is to generalize these kind of results to PDEs whose coefficient $\sigma$ depends on $\nabla u$, which is completely new as far as we know. Our motivation comes from image analysis. Indeed, let us consider a noisy image. The most basic way to smooth this 
image is to convolute it with a Gaussian kernel whose standard variation is a parameter $t$. If we represent the original noisy image by a continuous function $f:[0, N]^{2} \rightarrow \mathbb{R}$, the result of the convolution is

$$
g(t, x, y)=\frac{1}{2 \pi t^{2}} \iint_{\mathbb{R}^{2}} \exp \left(-\left(u^{2}+v^{2}\right) / 2 t^{2}\right) f(x+u, y+v) d u d v
$$

or in probabilistic terms by

$$
g(t, x, y)=E_{(x, y)}\left(f\left(B_{t}\right)\right)
$$

where $\left(B_{t}, t \geq 0\right)$ a planar Brownian motion starting from $(x, y)$.

Therefore $\mathrm{g}$ is the solution of the heat equation:

$$
\left\{\begin{array}{l}
\frac{\partial U}{\partial t}=\frac{1}{2} \Delta U \\
U(0, x, y)=f(x, y) .
\end{array}\right.
$$

This methods makes the noise disappear but its major drawback is that it also smooths edges. In order to take into account this problem and attenuate it, Perrona and Malik [7] suggest to study the next problem:

$$
\left\{\begin{array}{l}
\frac{\partial U}{\partial t}(t, x, y)=\operatorname{div}(\phi(|\nabla U(t, x, y)|) \nabla U(t, x, y)), \\
U(0, x, y)=f(x, y),
\end{array}\right.
$$

with, for instance, $\phi$ a Gaussian density. This has led to the so-called anisotropic diffusions. The main idea of these methods is to smooth the image in a direction parallel to the edges in order not to degrade them or in other words in the orthogonal direction of the gradient of the image. Consequently, all the coefficients of the PDEs that appear in these studies depend on the gradient of the solution.

In order to get a gradient in the second order term, we must make $\sigma$ depend on $z$. This case is partially studied in [6] concerning existence and uniqueness of the solution of the FBSDE but nothing else is done for that case. In the next section, we thus study a general FBSDE with $\sigma$ that depends also on $z$. We give another proof of existence and uniqueness of the solution on a small interval of time. We also focus on the regularity of the solution with respect to the initial conditions. In particular, we prove that the function $u(t, x)=Y_{t}^{t, x}$ is still continuous in that general case.

Then, we study the links between FBSDEs and PDEs. Here, we cannot deal with a general equation. Our result only applies for functions $f$ and $g$ that do not depend on $z$ when $\sigma$ does. We prove that, given a quasi-linear PDE, we may, under some additional assumptions, find a FBSDE such that the function $u(t, x)$ is a viscosity solution of the PDE.

Unfortunately, these additional assumptions are not very easy to verify and, in sections 4 and 5, we give sufficient conditions in dimension 1 and 2 for these assumptions to be satisfied.

Eventually, we propose in the last section another kind of FBSDE that would give an easier representation of the solution, avoiding the additional assumptions mentioned before. However, the techniques used in this paper cannot be directly extended to this case.

\section{Regularity OF THE SOLUTION WITH RESPECT TO INITIAL CONDITIONS}

2.1. Preliminaries. Let us first recall two well-known results: 
Theorem 2.1. (Perron-Frobenius)

If $A$ is a square matrix of order $n \geq 2$ with positive entries, then

- The spectral radius $\rho(A)$ is a real positive eigenvalue.

- The associated subspace has dimension 1 and is generated by a vector with positive components.

Theorem 2.2. (Picard fixed point theorem)

Let $E$ be a Banach space and let $f$ be a contraction on $E$ (i.e. a Lipschitz function with Lipschitz coefficient strictly less than 1). We define the sequence $\left(x_{n}\right)_{n \in \mathbb{N}}$ of elements of $E$ recursively by

$$
\left\{\begin{array}{l}
x_{0} \in E \\
x_{n+1}=f\left(x_{n}\right) \quad \forall n \in \mathbb{N}
\end{array}\right.
$$

Then

- The sequence $\left(x_{n}\right)_{n \in \mathbb{N}}$ converges in $E$.

- The limit $\ell$ of $\left(x_{n}\right)_{n \in \mathbb{N}}$ is the unique fixed point of $f$.

- For every $n \in \mathbb{N}$, we have

$$
\left\|x_{n}-x_{0}\right\| \leq \frac{1-c^{n}}{1-c}\left\|x_{1}-x_{0}\right\|
$$

where $c$ is the Lipschitz coefficient of $f$.

In particular, we have

$$
\left\|x_{0}-\ell\right\| \leq \frac{1}{1-c}\left\|x_{1}-x_{0}\right\| .
$$

We deduce from the Perron-Frobenius theorem another useful result: let $n$ be a fixed integer greater than 2. For every $i \in\{1,2, \ldots, n\}$, let $\mathcal{E}_{i}$ be a Banach space if endowed with the norm $N_{i}$. We denote by $\mathcal{E}$ the space product $\mathcal{E}_{1} \times \cdots \times \mathcal{E}_{n}$.

Definition 2.3. Let $f$ be a map from $\mathcal{E}$ to $\mathcal{E}$ :

$$
\begin{aligned}
f: \mathcal{E} & \longrightarrow \mathcal{E} \\
x & \longmapsto\left(f_{1}(x), f_{2}(x), \ldots, f_{n}(x)\right) .
\end{aligned}
$$

A matrix $A=\left(a_{i j}\right)_{1 \leq i, j \leq n}$ is said to be a Lipschitz matrix of $f$ if, for every $1 \leq j \leq n$ and every $x=\left(x_{1}, \ldots, x_{n}\right) \in \mathcal{E}$ and $y=\left(y_{1}, \ldots, y_{n}\right) \in \mathcal{E}$, we have

$$
N_{j}\left(f_{j}\left(x_{1}, \ldots, x_{n}\right)-f_{j}\left(y_{1}, \ldots, y_{n}\right)\right) \leq \sum_{i=1}^{n} a_{i j} N_{i}\left(x_{i}-y_{i}\right) .
$$

Theorem 2.4. Let $A$ be a Lipschitz matrix of $f$. If every entry of $A$ is positive then there exist positive constants $u_{1}, \ldots, u_{n}$ such that $f$ is Lipschitz with Lipschitz constant $\rho(A)$ with respect to the norm

$$
N\left(x_{1}, \ldots, x_{n}\right)=\sum_{i=1}^{n} u_{i} N_{i}\left(x_{i}\right) .
$$

Remark: Endowed with the norm $N, \mathcal{E}$ is a Banach space.

Proof: As the entries of $A$ are supposed to be positive, we can apply Perron-Frobenius theorem. Consequently we know that $\rho(A)$ is a real eigenvalue of $A$ and that there exists an 
eigenvector $\left(u_{1}, \ldots, u_{n}\right)$ with positive components. So, we set

$$
N\left(x_{1}, \ldots, x_{n}\right)=\sum_{i=1}^{n} u_{i} N_{i}\left(x_{i}\right) .
$$

By definition of a Lipschitz matrix, we have, for $x=\left(x_{1}, \ldots, x_{n}\right)$ and $y=\left(y_{1}, \ldots, y_{n}\right)$,

$$
\begin{aligned}
N(f(x)-f(y)) & =\sum_{j=1}^{n} u_{j} N_{j}\left(f_{j}\left(x_{1}, \ldots, x_{n}\right)-f_{j}\left(y_{1}, \ldots, y_{n}\right)\right) \\
& \leq \sum_{j=1}^{n} u_{j} \sum_{i=1}^{n} a_{i j} N_{i}\left(x_{i}-y_{i}\right) \\
& =\sum_{i=1}^{n} N_{i}\left(x_{i}-y_{i}\right) \sum_{j=1}^{n} a_{i j} u_{j} \\
& =\sum_{i=1}^{n} N_{i}\left(x_{i}-y_{i}\right) \rho(A) u_{i} \\
& =\rho(A) N(x-y)
\end{aligned}
$$

2.2. Existence and uniqueness of solutions of coupled FBSDE. Let $(\Omega, \mathcal{F}, \mathbb{P})$ be a probability space, $T>0$ and $\left(B_{t}, t \geq 0\right)$ be a $d$-dimensional Brownian motion. We set $\left(\mathcal{F}_{t}, t \geq 0\right)$ the completed natural filtration of $B$, and we denote by $\mathcal{M}^{2}(\mathcal{E})$ the set of $\mathcal{E}$-valued processes $\left(X_{t}, 0 \leq t \leq T\right)$ progressively measurable with respect to the filtration $\left(\mathcal{F}_{t}, t \geq 0\right)$ and such that

$$
\mathbb{E}\left[\int_{0}^{T}\left|X_{s}\right|^{2} d s\right]<+\infty
$$

We denote by $\mathcal{M}_{c}^{2}(\mathcal{E})$ the subset of $\mathcal{M}^{2}(\mathcal{E})$ of continuous processes $\left(X_{t}, 0 \leq t \leq T\right)$ such that

$$
\mathbb{E}\left[\sup _{t \in[0, T]}\left|X_{t}\right|^{2}\right]<+\infty .
$$

Here, the notation $|\cdot|$ refers to the absolute value, the Euclidean norm or the square root of the sum of squares of the components respectively when the content is a real number, a vector or a matrix.

We consider here a general FBSDE. Let us first fix the following functions :

$$
\begin{aligned}
& \sigma: \Omega \times[0,+\infty) \times \mathbb{R}^{d} \times \mathbb{R} \times \mathbb{R}^{d} \longmapsto \mathbb{R}^{d \times d}, \\
& f: \Omega \times[0,+\infty) \times \mathbb{R}^{d} \times \mathbb{R} \times \mathbb{R}^{d} \longmapsto \mathbb{R}^{d}, \\
& g: \Omega \times[0,+\infty) \times \mathbb{R}^{d} \times \mathbb{R} \times \mathbb{R}^{d} \longmapsto \mathbb{R}, \\
& h: \Omega \times \mathbb{R}^{d} \longmapsto \mathbb{R} .
\end{aligned}
$$

We are studying the following FBSDE

$$
\begin{cases}X_{s}=x+\int_{0}^{s} f\left(r, X_{r}, Y_{r}, Z_{r}\right) d r+\int_{0}^{s} \sigma\left(r, X_{r}, Y_{r}, Z_{r}\right) d B_{r}, & 0 \leq s \leq T, \\ Y_{s}=h\left(X_{T}\right)+\int_{s}^{T} g\left(r, X_{r}, Y_{r}, Z_{r}\right) d r-\int_{s}^{T}\left\langle Z_{r}, d B_{r}\right\rangle, & 0 \leq s \leq T,\end{cases}
$$


for which we are seeking solution $\left\{\left(X_{s}, Y_{s}, Z_{s}\right), 0 \leq s \leq T\right\}$ that belongs to $\mathcal{M}_{c}^{2}\left(\mathbb{R}^{d}\right) \times$ $\mathcal{M}_{c}^{2}(\mathbb{R}) \times \mathcal{M}^{2}\left(\mathbb{R}^{d}\right)$. Here, $<\cdot, \cdot>$ denotes the usual scalar product in $\mathbb{R}^{d}$.

Some conditions for existence and uniqueness of solutions of the FBSDE (2) have been given in [6]. We will study here a Picard iteration that leads to other conditions for existence and uniqueness of the solutions as a by-product but the purpose of this subsection is the final Theorem 2.7.

We will suppose that the functions $f, g, h$ and $\sigma$ satisfy the following properties:

[H1] For $\phi=f, g, \sigma$, there exist non-negative constants $k_{\phi, x}, k_{\phi, y}, k_{\phi, z}$ such that, for all $t, x, x^{\prime}, y, y^{\prime}, z, z^{\prime}$ and a.s.,

$$
\left|\phi(t, x, y, z)-\phi\left(t, x^{\prime}, y^{\prime}, z^{\prime}\right)\right| \leq k_{\phi, x}\left|x-x^{\prime}\right|+k_{\phi, y}\left|y-y^{\prime}\right|+k_{\phi, z}\left|z-z^{\prime}\right| .
$$

[H2] There exists a non-negative constant $k_{h}$ such that, for all $x, x^{\prime}$ and a.s.,

$$
\left|h(x)-h\left(x^{\prime}\right)\right| \leq k_{h}\left|x-x^{\prime}\right|
$$

[H3] For all $x, y, z$, the processes $f(\cdot, x, y, z), g(\cdot, x, y, z)$ and $\sigma(\cdot, x, y, z)$ are progressively measurable with respect to the filtration $\left(\mathcal{F}_{t}, t \geq 0\right)$; for all $x$, the random variable $h(x)$ is $\mathcal{F}_{T}$-measurable and

$$
\mathbb{E}\left[\int_{0}^{T}|f(r, 0,0,0)|^{2} d r+\int_{0}^{T}|g(r, 0,0,0)|^{2} d r+\int_{0}^{T}|\sigma(r, 0,0,0)|^{2} d r+|h(0)|^{2}\right]<+\infty .
$$

Under these conditions, we may consider the map $\Psi$ defined by:

$$
\begin{aligned}
\Psi: \mathcal{M}_{c}^{2}\left(\mathbb{R}^{d}\right) \times \mathcal{M}_{c}^{2}(\mathbb{R}) \times \mathcal{M}^{2}\left(\mathbb{R}^{d}\right) & \longrightarrow \mathcal{M}_{c}^{2}\left(\mathbb{R}^{d}\right) \times \mathcal{M}_{c}^{2}(\mathbb{R}) \times \mathcal{M}^{2}\left(\mathbb{R}^{d}\right) \\
(U, V, W) & \longrightarrow(X, Y, Z)
\end{aligned}
$$

where, for every $0 \leq t \leq T$,

$$
\begin{aligned}
& X_{t}=x+\int_{0}^{t} f\left(s, U_{s}, V_{s}, W_{s}\right) d s+\int_{0}^{t} \sigma\left(s, U_{s}, V_{s}, W_{s}\right) d B_{s} \\
& Y_{t}=\mathbb{E}\left[h\left(X_{T}\right) \mid \mathcal{F}_{t}\right]+\mathbb{E}\left[\int_{t}^{T} g\left(u, U_{u}, V_{u}, W_{u}\right) d u \mid \mathcal{F}_{t}\right]
\end{aligned}
$$

and $\left(Z_{t}, 0 \leq t \leq T\right)$ is the unique element of $\mathcal{M}^{2}\left(\mathbb{R}^{d}\right)$ such that

$$
h\left(X_{T}\right)+\int_{0}^{T} g\left(u, U_{u}, V_{u}, W_{u}\right) d u-Y_{0}=\int_{0}^{T}\left\langle Z_{s}, d B_{s}\right\rangle .
$$

In other words, $(X, Y, Z)$ is solution of the system

$$
\begin{cases}X_{t}=x+\int_{0}^{t} f\left(s, U_{s}, V_{s}, W_{s}\right) d s+\int_{0}^{t} \sigma\left(s, U_{s}, V_{s}, W_{s}\right) d B_{s}, & 0 \leq t \leq T, \\ Y_{t}=h\left(X_{T}\right)+\int_{t}^{T} g\left(s, U_{s}, V_{s}, W_{s}\right) d s-\int_{t}^{T}<Z_{s}, d B_{s}>, & 0 \leq t \leq T .\end{cases}
$$

Let us remark that a solution of the FBSDE (2) is a fixed point of $\Psi$.

For $X \in \mathcal{M}_{c}^{2}(\mathcal{E}), \mathcal{E}=\mathbb{R}$ or $\mathcal{E}=\mathbb{R}^{d}$, let us denote by

$$
\|X\|_{c}=\sqrt{\mathbb{E}\left[\sup _{0 \leq s \leq t}\left|X_{s}\right|^{2}\right]}
$$

Endowed with this norm, $\mathcal{M}_{c}^{2}(\mathcal{E})$ is a Banach space. 
For $X \in \mathcal{M}^{2}(\mathcal{E})$, we denote

$$
\|X\|_{2}=\left(\int_{0}^{T} \mathbb{E}\left[\left|X_{s}\right|^{2}\right] d s\right)^{1 / 2}
$$

and $\mathcal{M}^{2}(\mathcal{E})$ is also a Banach space with respect to this norm.

Proposition 2.5. Let us suppose that the assumptions [H1], [H2], [H3] are satisfied. The matrix $A(T)$ defined by

$$
\sqrt{6}\left(\begin{array}{ccc}
\sqrt{T} \sqrt{T k_{f, x}^{2}+4 k_{\sigma, x}^{2}} & 2 \sqrt{T} \sqrt{8 k_{h}^{2} k_{\sigma, x}^{2}+T\left(k_{g, x}^{2}+2 k_{h}^{2} k_{f, x}^{2}\right)} & \sqrt{T} \sqrt{T\left(k_{g, x}^{2}+2 k_{h}^{2} k_{f, x}^{2}\right)+8 k_{h}^{2} k_{\sigma, x}^{2}} \\
\sqrt{T} \sqrt{T k_{f, y}^{2}+4 k_{\sigma, y}^{2}} & 2 \sqrt{T} \sqrt{8 k_{h}^{2} k_{\sigma, y}^{2}+T\left(k_{g, y}^{2}+2 k_{h}^{2} k_{f, y}^{2}\right)} & \sqrt{T} \sqrt{T\left(k_{g, y}^{2}+2 k_{h}^{2} k_{f, y}^{2}\right)+8 k_{h}^{2} k_{\sigma, y}^{2}} \\
\sqrt{T k_{f, z}^{2}+4 k_{\sigma, z}^{2}} & 2 \sqrt{8 k_{h}^{2} k_{\sigma, z}^{2}+T\left(2 k_{h}^{2} k_{f, z}^{2}+k_{g, z}^{2}\right)} & \sqrt{T\left(k_{g, z}^{2}+2 k_{h}^{2} k_{f, z}^{2}\right)+8 k_{h}^{2} k_{\sigma, z}^{2}}
\end{array}\right)
$$

is a Lipschitz matrix for $\Psi$.

Proof: Let $(U, V, W) \in \mathcal{M}_{c}^{2}\left(\mathbb{R}^{d}\right) \times \mathcal{M}_{c}^{2}(\mathbb{R}) \times \mathcal{M}^{2}\left(\mathbb{R}^{d}\right)$ and $\left(U^{\prime}, V^{\prime}, W^{\prime}\right) \in \mathcal{M}_{c}^{2}\left(\mathbb{R}^{d}\right) \times \mathcal{M}_{c}^{2}(\mathbb{R}) \times$ $\mathcal{M}^{2}\left(\mathbb{R}^{d}\right)$. We denote

$$
\begin{aligned}
(X, Y, Z) & =\Psi(U, V, W) \\
\left(X^{\prime}, Y^{\prime}, Z^{\prime}\right) & =\Psi\left(U^{\prime}, V^{\prime}, W^{\prime}\right) \\
\Delta X & =X-X^{\prime} \\
\Delta Y & =Y-Y^{\prime} \\
\Delta Z & =Z-Z^{\prime} \\
\Delta U & =U-U^{\prime} \\
\Delta V & =V-V^{\prime} \\
\Delta W & =W-W^{\prime} .
\end{aligned}
$$

First, let us estimate $\|\Delta X\|_{c}$. For $0 \leq s \leq T$, we have

$$
\begin{aligned}
X_{s}-X_{s}^{\prime}= & \int_{0}^{s}\left[f\left(u, U_{u}, V_{u}, W_{u}\right)-f\left(u, U_{u}^{\prime}, V_{u}^{\prime}, W_{u}^{\prime}\right)\right] d u \\
& \quad+\int_{0}^{s}\left[\sigma\left(u, U_{u}, V_{u}, W_{u}\right)-\sigma\left(u, U_{u}^{\prime}, V_{u}^{\prime}, W_{u}^{\prime}\right)\right] d B_{u} \\
:= & \int_{0}^{s} \Delta f_{u} d u+\int_{0}^{s} \Delta \sigma_{u} d B_{u} .
\end{aligned}
$$

Using the inequality $(a+b)^{2} \leq 2\left(a^{2}+b^{2}\right)$, we obtain

$$
\|\Delta X\|_{c}^{2} \leq 2 \mathbb{E}\left[\sup _{0 \leq s \leq T}\left(\int_{0}^{s}\left|\Delta f_{u}\right| d u\right)^{2}\right]+2 \mathbb{E}\left[\sup _{0 \leq s \leq T}\left|\int_{0}^{s} \Delta \sigma_{u} d B_{u}\right|^{2}\right] .
$$

We then apply Cauchy-Schwartz inequality for the first term and Doob's inequality for the second to get

$$
\|\Delta X\|_{c}^{2} \leq 2 T \mathbb{E}\left[\int_{0}^{T}\left|\Delta f_{u}\right|^{2} d u\right]+8 \mathbb{E}\left[\int_{0}^{T}\left|\Delta \sigma_{u}\right|^{2} d u\right] .
$$


But, by assumption [H1],

$$
\begin{aligned}
& \left|\Delta f_{u}\right|^{2} \leq 3 k_{f, x}^{2}\left|\Delta U_{u}\right|^{2}+3 k_{f, y}^{2}\left|\Delta V_{u}\right|^{2}+3 k_{f, z}^{2}\left|\Delta W_{u}\right|^{2}, \\
& \left|\Delta \sigma_{u}\right|^{2} \leq 3 k_{\sigma, x}^{2}\left|\Delta U_{u}\right|^{2}+3 k_{\sigma, y}^{2}\left|\Delta V_{u}\right|^{2}+3 k_{\sigma, z}^{2}\left|\Delta W_{u}\right|^{2} .
\end{aligned}
$$

Consequently, we obtain

$$
\begin{aligned}
\|\Delta X\|_{c}^{2} \leq\left(6 T k_{f, x}^{2}+24 k_{\sigma, x}^{2}\right) \mathbb{E}\left[\int_{0}^{T}\left|\Delta U_{u}\right|^{2} d u\right]+\left(6 T k_{f, y}^{2}+24 k_{\sigma, y}^{2}\right) \mathbb{E}\left[\int_{0}^{T}\left|\Delta V_{u}\right|^{2} d u\right] \\
+\left(6 T k_{f, z}^{2}+24 k_{\sigma, z}^{2}\right) \mathbb{E}\left[\int_{0}^{T}\left|\Delta W_{u}\right|^{2} d u\right] \\
\leq 6 T\left(T k_{f, x}^{2}+4 k_{\sigma, x}^{2}\right)\|\Delta U\|_{c}^{2}+6 T\left(T k_{f, y}^{2}+4 k_{\sigma, y}^{2}\right)\|\Delta V\|_{c}^{2}+6\left(T k_{f, z}^{2}+4 k_{\sigma, z}^{2}\right)\|\Delta W\|_{2}^{2} .
\end{aligned}
$$

The first column of the matrix follows then easily.

Let us concentrate now on the estimate for $\|\Delta Y\|_{c}$. By definition of $Y$, we have for $0 \leq s \leq T$, with obvious notations,

$$
\Delta Y_{s}=\mathbb{E}\left[h\left(X_{T}\right)-h\left(X_{T}^{\prime}\right) \mid \mathcal{F}_{s}\right]+\mathbb{E}\left[\int_{s}^{T} \Delta g_{u} d u \mid \mathcal{F}_{s}\right]
$$

which yields to

$$
\sup _{0 \leq s \leq T}\left|\Delta Y_{s}\right|^{2} \leq 2\left(\sup _{0 \leq s \leq T} \mathbb{E}\left[\left|h\left(X_{T}\right)-h\left(X_{T}^{\prime}\right)\right| \mid \mathcal{F}_{s}\right]^{2}+\sup _{0 \leq s \leq T} \mathbb{E}\left[\int_{s}^{T}\left|\Delta g_{u}\right| d u \mid \mathcal{F}_{s}\right]^{2}\right) .
$$

As the process $\left(\mathbb{E}\left[\left|h\left(X_{T}\right)-h\left(X_{T}^{\prime}\right)\right| \mid \mathcal{F}_{s}\right], 0 \leq s \leq T\right)$ is a martingale, we have by Doob's inequality

$$
\begin{aligned}
\mathbb{E}\left[\sup _{0 \leq s \leq T} \mathbb{E}\left[\left|h\left(X_{T}\right)-h\left(X_{T}^{\prime}\right)\right| \mid \mathcal{F}_{s}\right]^{2}\right] & \leq 4 \sup _{0 \leq s \leq T} \mathbb{E}\left[\mathbb{E}\left[\left|h\left(X_{T}\right)-h\left(X_{T}^{\prime}\right)\right| \mid \mathcal{F}_{s}\right]^{2}\right] \\
& \leq 4 \mathbb{E}\left[\left|h\left(X_{T}\right)-h\left(X_{T}^{\prime}\right)\right|^{2}\right]
\end{aligned}
$$

by Jensen's inequality.

By similar arguments, we have

$$
\begin{aligned}
\mathbb{E}\left[\sup _{0 \leq s \leq T} \mathbb{E}\left[\int_{s}^{T}\left|\Delta g_{u}\right| d u \mid \mathcal{F}_{s}\right]^{2}\right] & \leq \mathbb{E}\left[\sup _{0 \leq s \leq T} \mathbb{E}\left[\int_{0}^{T}\left|\Delta g_{u}\right| d u \mid \mathcal{F}_{s}\right]^{2}\right] \\
& \leq 4 \mathbb{E}\left[\left(\int_{0}^{T}\left|\Delta g_{u}\right| d u\right)^{2}\right] \\
& \leq 4 T \mathbb{E}\left[\int_{0}^{T}\left|\Delta g_{u}\right|^{2} d u\right]
\end{aligned}
$$

by Cauchy-Schwartz inequality. So, using assumptions [H1] for $\Delta g$ and [H2], we have

$$
\begin{aligned}
\|\Delta Y\|_{c}^{2} & \leq 8 \mathbb{E}\left[\left|h\left(X_{T}\right)-h\left(X_{T}^{\prime}\right)\right|^{2}\right]+8 T \mathbb{E}\left[\int_{0}^{T}\left|\Delta g_{u}\right|^{2} d u\right] \\
& \leq 8 k_{h}^{2}\|\Delta X\|_{c}^{2}+24 T \mathbb{E}\left[\int_{0}^{T}\left(k_{g, x}^{2}\left|\Delta U_{u}\right|^{2}+k_{g, y}^{2}\left|\Delta V_{u}\right|^{2}+k_{g, z}^{2}\left|\Delta W_{u}\right|^{2}\right) d u\right] .
\end{aligned}
$$


Plugging into the last estimates the upper bound for $\|\Delta X\|_{c}^{2}$ leads to the second column of the matrix.

Eventually, we have for $\Delta Z$

$$
\begin{aligned}
\|\Delta Z\|_{2}^{2} & =\mathbb{E}\left[\left|\int_{0}^{T}<\Delta Z_{u}, d B_{u}>\right|^{2}\right] \\
& =\mathbb{E}\left[\left|h\left(X_{T}\right)-h\left(X_{T}^{\prime}\right)-\mathbb{E}\left[h\left(X_{T}\right)-h\left(X_{T}^{\prime}\right)\right]+\int_{0}^{T} \Delta g_{u} d u-\mathbb{E}\left[\int_{0}^{T} \Delta g_{u} d u\right]\right|^{2}\right] \\
& \leq 2 \mathbb{E}\left[\left|h\left(X_{T}\right)-h\left(X_{T}^{\prime}\right)\right|^{2}\right]+2 \mathbb{E}\left[\left|\int_{0}^{T} \Delta g_{u} d u\right|^{2}\right] \\
& \leq 2 k_{h}^{2}\|\Delta X\|_{c}^{2}+2 T \mathbb{E}\left[\int_{0}^{T}\left|\Delta g_{u}\right|^{2} d u\right] \\
& \leq 2 k_{h}^{2}\|\Delta X\|_{c}^{2}+6 T \mathbb{E}\left[\int_{0}^{T}\left(k_{g, x}^{2}\left|\Delta U_{u}\right|^{2}+k_{g, y}^{2}\left|\Delta V_{u}\right|^{2}+k_{g, z}^{2}\left|\Delta W_{u}\right|^{2}\right) d u\right] .
\end{aligned}
$$

and the third column of the matrix follows easily.

Let us remark that the matrix $A(T)$ becomes, for $T=0$,

$$
A(0)=\sqrt{6}\left(\begin{array}{ccc}
0 & 0 & 0 \\
0 & 0 & 0 \\
2 k_{\sigma, z} & 4 \sqrt{2} k_{h} k_{\sigma, z} & \sqrt{8} k_{h} k_{\sigma, z}
\end{array}\right)
$$

whose spectral radius is $4 \sqrt{3} k_{h} k_{\sigma, z}$. We may deduce then the following result:

Theorem 2.6. If the assumptions [H1], [H2], [H3] are satisfied and if moreover

$$
4 \sqrt{3} k_{h} k_{\sigma, z}<1
$$

then the FBSDE (2) has a unique solution if $T$ is small enough.

Proof: We want to apply Theorem 2.4 to the map $\Psi$. As we need a Lipschitz matrix with positive entries, let us set, for $\varepsilon>0$,

$$
A^{\varepsilon}(T)=A(T)+\varepsilon\left(\begin{array}{lll}
1 & 1 & 1 \\
1 & 1 & 1 \\
1 & 1 & 1
\end{array}\right) .
$$

As the matrix $A(T)$ has its entries non-negative, the entries of $A^{\varepsilon}(T)$ are positive and $A^{\varepsilon}(T)$ is still a Lipschitz matrix of $\Psi$. Moreover, as the spectral radius is continuous, we may fix $T$ small enough and $\varepsilon>0$ such that $\rho\left(A^{\varepsilon}(T)\right)<1$. Then, Theorem 2.4 gives that there exists a norm on $\mathcal{M}^{2}\left(\mathbb{R}^{d}\right) \times \mathcal{M}_{c}^{2}(\mathbb{R}) \times \mathcal{M}^{2}\left(\mathbb{R}^{d}\right)$ for which $\Psi$ is a contraction and, by Picard theorem, $\Psi$ has a unique fixed point.

Remark: we may also fix the terminal time $T$ and, as $A$ tends to the null matrix as the Lipschitz coefficients tend to 0, we have, by the same kind of arguments, existence and uniqueness of the solution on the interval $[0, T]$ if the Lipschitz coefficients are small enough.

As announced before, the purpose of this section is not to prove existence and uniqueness of the solutions (which have already been proved) but the use of the Lipschitz matrix leads to some estimates in the same spirit of [6], Theorem 4.1. 
Theorem 2.7. Let us suppose that the assumptions [H1], [H2], [H3] and (3) are satisfied. Let $T>0$ be such that there exists a unique adapted solution $\left(X_{s}, Y_{s}, Z_{s}\right)$ to the system (2). Then, there exists a positive constant $C$ such that

$$
\begin{aligned}
& \mathbb{E}\left[\sup _{0 \leq s \leq T}\left|X_{s}\right|^{2}\right]+\mathbb{E}\left[\sup _{0 \leq s \leq T}\left|Y_{s}\right|^{2}\right]+\mathbb{E}\left[\int_{0}^{T}\left|Z_{s}\right|^{2} d s\right] \\
\leq & C\left(|x|^{2}+\mathbb{E}\left[\int_{0}^{T}|f(s, 0,0,0)|^{2} d s+\int_{0}^{T}|g(s, 0,0,0)|^{2} d s+\int_{0}^{T}|\sigma(s, 0,0,0)|^{2} d s+|h(0)|^{2}\right]\right) .
\end{aligned}
$$

Proof: We still consider the matrix $A^{\varepsilon}(T)$ for some $\varepsilon>0$ small enough such that $\rho\left(A^{\varepsilon}(T)\right)<1$. Then, by Theorem 2.4, there exist positive constants $\alpha, \beta, \gamma$ such that, if we consider the norm

then

$$
N(x, y, z)=\alpha\|x\|_{c}+\beta\|y\|_{c}+\gamma\|z\|_{2},
$$

$$
N\left(\left(X_{s}, 0 \leq s \leq T\right),\left(Y_{s}, 0 \leq s \leq T\right),\left(Z_{s}, 0 \leq s \leq T\right)\right) \leq \frac{N(\Psi(0,0,0))}{1-\rho\left(A^{\varepsilon}(T)\right)} .
$$
that

But, by the same computations as in the proof of Proposition 2.5, there exists $K>0$ such

$$
\begin{array}{r}
N(\Psi(0,0,0))^{2} \leq \sup (\alpha, \beta, \gamma)^{2} K\left(|x|^{2}+\mathbb{E}\left[\int_{0}^{T}|f(s, 0,0,0)|^{2} d s+\int_{0}^{T}|g(s, 0,0,0)|^{2} d s\right]\right. \\
\left.+\mathbb{E}\left[\int_{0}^{T}|\sigma(s, 0,0,0)|^{2} d s\right]+|h(0)|^{2}\right) .
\end{array}
$$

The result then follows easily with

$$
C=\frac{K \sup (\alpha, \beta, \gamma)^{2}}{\left(1-\rho\left(A^{\varepsilon}(T)\right)\right) \inf (\alpha, \beta, \gamma)^{2}} .
$$

2.3. Regularity of the solution with respect to initial conditions. Let us now consider the solution $\left(\left(X_{s}^{t, x}, Y_{s}^{t, x}, Z_{s}^{t, x}\right), t \leq s \leq T\right)$ that corresponds to the initial point $(t, x)$ for $X$. In other words, $\left(\left(X_{s}^{t, x}, Y_{s}^{t, x}, Z_{s}^{t, x}\right), t \leq s \leq T\right)$ is the solution of the following FBSDE:

$$
\left\{\begin{array}{l}
X_{s}=x+\int_{t}^{s} f\left(r, X_{r}, Y_{r}, Z_{r}\right) d r+\int_{t}^{s} \sigma\left(r, X_{r}, Y_{r}, Z_{r}\right) d B_{r}, t \leq s \leq T, \\
Y_{s}=h\left(X_{T}\right)+\int_{s}^{T} g\left(r, X_{r}, Y_{r}, Z_{r}\right) d r-\int_{s}^{T}\left\langle Z_{r}, d B_{r}\right\rangle, t \leq s \leq T .
\end{array}\right.
$$

We suppose that the conditions [H1], [H2], [H3] and (3) are satisfied so that there is a unique adapted (to the filtration generated by $\left.\left(\left(B_{s}-B_{t}\right), s \geq t\right)\right)$ solution to (4) for $T$ small enough. Moreover, we consider in this section only deterministic functions $f, g, h$ and $\sigma$.

Then, by Blumenthal zero-one law, the variable $Y_{t}^{t, x}$ is deterministic.

Theorem 2.8. Under these assumptions, the function $u:[0, T] \times \mathbb{R}^{d} \longrightarrow \mathbb{R}$ defined by $u(t, x)=Y_{t}^{t, x}$ is continuous on $[0, T] \times \mathbb{R}^{d}$.

Proof: Let $\left(t_{0}, x_{0}\right) \in[0, T] \times \mathbb{R}^{d}$. For any $(t, x) \in[0, T] \times \mathbb{R}^{d}$, let us set

$$
\begin{aligned}
& \hat{f}_{t}(r, x, y, z)=\mathbf{1}_{r \geq t} f(r, x, y, z) \\
& \hat{\sigma}_{t}(r, x, y, z)=\mathbf{1}_{r \geq t} \sigma(r, x, y, z) \\
& \hat{g}_{t}(r, x, y, z)=\mathbf{1}_{r \geq t} g(r, x, y, z)
\end{aligned}
$$


and let us consider $\left(\left(\hat{X}_{s}^{t, x}, \hat{Y}_{s}^{t, x}, \hat{Z}_{s}^{t, x}\right), 0 \leq s \leq T\right)$ solution of the system

$$
\left\{\begin{array}{l}
\hat{X}_{s}^{t, x}=x+\int_{0}^{s} \hat{f}_{t}\left(u, \hat{X}_{u}^{t, x}, \hat{Y}_{u}^{t, x}, \hat{Z}_{u}^{t, x}\right) d u+\int_{0}^{s} \hat{\sigma}_{t}\left(u, \hat{X}_{u}^{t, x}, \hat{Y}_{u}^{t, x}, \hat{Z}_{u}^{t, x}\right) d B_{u}, \\
\hat{Y}_{s}^{t, x}=h\left(\hat{X}_{T}^{t, x}\right)+\int_{s}^{T} \hat{g}_{t}\left(u, \hat{X}_{u}^{t, x}, \hat{Y}_{u}^{t, x}, \hat{Z}_{u}^{t, x}\right) d u-\int_{s}^{T}<\hat{Z}_{u}^{t, x} d B_{u}>.
\end{array}\right.
$$

Let us note that

$$
\begin{cases}\hat{X}_{s}^{t, x}=x & \text { for } 0 \leq s<t \\ \hat{X}_{s}^{t, x}=X_{s}^{t, x} & \text { for } t \leq s \leq T,\end{cases}
$$

that

$$
\begin{cases}\hat{Y}_{s}^{t, x}=Y_{t}^{t, x} & \text { for } 0 \leq s<t \\ \hat{Y}_{s}^{t, x}=Y_{s}^{t, x} & \text { for } t \leq s \leq T\end{cases}
$$

and that

$$
\begin{cases}\hat{Z}_{s}^{t, x}=0 & \text { for } 0 \leq s<t \\ \hat{Z}_{s}^{t, x}=Z_{s}^{t, x} & \text { for } t \leq s \leq T .\end{cases}
$$

Hence, $\left(\hat{X}^{t, x}, \hat{Y}^{t, x}, \hat{Z}^{t, x}\right)$ may be viewed as an extension of $\left(X^{t, x}, Y^{t, x}, Z^{t, x}\right)$ on $[0, T]$. Let us then define for $0 \leq s \leq T$,

$$
\bar{X}_{s}=\hat{X}_{s}^{t, x}-\hat{X}_{s}^{t_{0}, x_{0}}, \quad \bar{Y}_{s}=\hat{Y}_{s}^{t, x}-\hat{Y}_{s}^{t_{0}, x_{0}}, \quad \bar{Z}_{s}=\hat{Z}_{s}^{t, x}-\hat{Z}_{s}^{t_{0}, x_{0}} .
$$

$(\bar{X}, \bar{Y}, \bar{Z})$ is then solution of the system

$$
\left\{\begin{array}{l}
\bar{X}_{s}=x-x_{0}+\int_{0}^{s} \bar{f}\left(u, \bar{X}_{u}, \bar{Y}_{u}, \bar{Z}_{u}\right) d u+\int_{0}^{s} \bar{\sigma}\left(u, \bar{X}_{u}, \bar{Y}_{u}, \bar{Z}_{u}\right) d B_{u}, \\
\bar{Y}_{s}=\bar{h}\left(\bar{X}_{T}\right)+\int_{s}^{T} \bar{g}\left(u, \bar{X}_{u}, \bar{Y}_{u}, \bar{Z}_{u}\right) d u-\int_{s}^{T}<\bar{Z}_{u}, d B_{u}>,
\end{array}\right.
$$

where $\bar{f}, \bar{g}, \bar{\sigma}$ and $\bar{h}$ are defined by

$$
\begin{aligned}
\bar{f}(u, x, y, z) & =\hat{f}_{t}\left(u, x+\hat{X}_{u}^{t_{0}, x_{0}}, y+\hat{Y}_{u}^{t_{0}, x_{0}}, z+\hat{Z}_{u}^{t_{0}, x_{0}}\right)-\hat{f}_{t_{0}}\left(u, \hat{X}_{u}^{t_{0}, x_{0}}, \hat{Y}_{u}^{t_{0}, x_{0}}, \hat{Z}_{u}^{t_{0}, x_{0}}\right), \\
\bar{g}(u, x, y, z) & =\hat{g}_{t}\left(u, x+\hat{X}_{u}^{t_{0}, x_{0}}, y+\hat{Y}_{u}^{t_{0}, x_{0}}, z+\hat{Z}_{u}^{t_{0}, x_{0}}\right)-\hat{g}_{t_{0}}\left(u, \hat{X}_{u}^{t_{0}, x_{0}}, \hat{Y}_{u}^{t_{0}, x_{0}}, \hat{Z}_{u}^{t_{0}, x_{0}}\right), \\
\bar{\sigma}(u, x, y, z) & =\hat{\sigma}_{t}\left(u, x+\hat{X}_{u}^{t_{0}, x_{0}}, y+\hat{Y}_{u}^{t_{0}, x_{0}}, z+\hat{Z}_{u}^{t_{0}, x_{0}}\right)-\hat{\sigma}_{t_{0}}\left(u, \hat{X}_{u}^{t_{0}, x_{0}}, \hat{Y}_{u}^{t_{0}, x_{0}}, \hat{Z}_{u}^{t_{0}, x_{0}}\right), \\
\bar{h}(x) & =h\left(x+\hat{X}_{T}^{t_{0}, x_{0}}\right)-h\left(\hat{X}_{T}^{t_{0}, x_{0}}\right) .
\end{aligned}
$$

As the functions $\bar{f}, \bar{g}, \bar{\sigma}$ and $\bar{h}$ also satisfy assumptions [H1], [H2], [H3] and (3) (with the same Lipschitz constants as $f, g, \sigma$ and $h$ respectively), we may apply Theorem 2.7 to $(\bar{X}, \bar{Y}, \bar{Z})$. Thus, there exists a constant $A>0$ such that

$$
\begin{aligned}
\mathbb{E}\left[\sup _{0 \leq s \leq T}\left|\bar{X}_{s}\right|^{2}\right]+\mathbb{E}\left[\sup _{0 \leq s \leq T}\left|\bar{Y}_{s}\right|^{2}\right]+\mathbb{E}\left[\int_{0}^{T}\left|\bar{Z}_{u}\right|^{2} d u\right] & \\
\leq A\left(\left|x-x_{0}\right|^{2}+\mathbb{E}\left[\int_{0}^{T}|\bar{f}(u, 0,0,0)|^{2} d u\right.\right. & +\int_{0}^{T}|\bar{g}(u, 0,0,0)|^{2} d u \\
& \left.\left.+\int_{0}^{T}|\bar{\sigma}(u, 0,0,0)|^{2} d u+|\bar{h}(0)|^{2}\right]\right)
\end{aligned}
$$


But,

$$
\int_{0}^{T}|\bar{f}(u, 0,0,0)|^{2} d u=\int_{t \wedge t_{0}}^{t \vee t_{0}}\left|f\left(u, \hat{X}_{u}^{t_{0}, x_{0}}, \hat{Y}_{u}^{t_{0}, x_{0}}, \hat{Z}_{u}^{t_{0}, x_{0}}\right)\right|^{2} d u
$$

and by assumption [H1], we have

$$
\int_{0}^{T}|\bar{f}(u, 0,0,0)|^{2} d u \leq C\left(\int_{t \wedge t_{0}}^{t \vee t_{0}}\left(|f(u, 0,0,0)|^{2}+\left|\hat{X}_{u}^{t_{0}, x_{0}}\right|^{2}+\left|\hat{Y}_{u}^{t_{0}, x_{0}}\right|^{2}+\left|\hat{Z}_{u}^{t_{0}, x_{0}}\right|^{2}\right) d u\right) .
$$

Then, applying again Theorem 2.7 to $\left(\hat{X}^{t_{0}, x_{0}}, \hat{Y}^{t_{0}, x_{0}}, \hat{Z}^{t_{0}, x_{0}}\right)$, we obtain that

$$
\mathbb{E}\left[\sup _{0 \leq s \leq T}\left|\hat{X}_{s}^{t_{0}, x_{0}}\right|^{2}\right]+\mathbb{E}\left[\sup _{0 \leq s \leq T}\left|\hat{Y}_{s}^{t_{0}, x_{0}}\right|^{2}\right]<+\infty .
$$

Thus we have proved that there exists a constant $C$ such that

$$
\mathbb{E}\left[\int_{0}^{T}|\bar{f}(u, 0,0,0)|^{2} d u\right] \leq C\left(\left|t-t_{0}\right|+\mathbb{E}\left[\int_{t \wedge t_{0}}^{t \vee t_{0}}\left(|f(u, 0,0,0)|^{2}+\left|\hat{Z}_{u}^{t_{0}, x_{0}}\right|^{2}\right) d u\right]\right) .
$$

The same reasoning also applies for $g$ and $\sigma$ and we get that there exists a constant $C$ such that

$$
\begin{aligned}
\mathbb{E}\left[\sup _{0 \leq s \leq T}\left|\bar{X}_{s}\right|^{2}\right]+ & \mathbb{E}\left[\sup _{0 \leq s \leq T}\left|\bar{Y}_{s}\right|^{2}\right]+ \\
\leq & \mathbb{E}\left[\int_{0}^{T}\left|\bar{Z}_{u}\right|^{2} d u\right] \\
\leq & \left(\left|x-x_{0}\right|^{2}+\left|t-t_{0}\right|+\mathbb{E}\left[\mid \int_{t_{0}}^{t}\left(|f(u, 0,0,0)|^{2}\right.\right.\right. \\
& \left.\left.\left.+|g(u, 0,0,0)|^{2}+|\sigma(u, 0,0,0)|^{2}+\left|\hat{Z}_{u}^{t_{0}, x_{0}}\right|^{2}\right) d u \mid\right]\right) .
\end{aligned}
$$

Let us now note that

$$
\begin{aligned}
\left|u(t, x)-u\left(t_{0}, x_{0}\right)\right|^{2}= & \mathbb{E}\left[\left|\hat{Y}_{t}^{t, x}-\hat{Y}_{t_{0}}^{t_{0}, x_{0}}\right|^{2}\right] \\
\leq & 2 \mathbb{E}\left[\left|\hat{Y}_{t}^{t, x}-\hat{Y}_{t}^{t_{0}, x_{0}}\right|^{2}\right]+2 \mathbb{E}\left[\left|\hat{Y}_{t}^{t_{0}, x_{0}}-\hat{Y}_{t_{0}}^{t_{0}, x_{0}}\right|^{2}\right] \\
\leq & C\left(\left|x-x_{0}\right|^{2}+\left|t-t_{0}\right|+\mathbb{E}\left[\mid \int_{t_{0}}^{t}\left(|f(u, 0,0,0)|^{2}\right.\right.\right. \\
& \left.\left.\left.\quad+|g(u, 0,0,0)|^{2}+|\sigma(u, 0,0,0)|^{2}+\left|\hat{Z}_{u}^{t_{0}, x_{0}}\right|^{2}\right) d u \mid\right]\right) .
\end{aligned}
$$

As

$$
\mathbb{E}\left[\left|\int_{0}^{T}\left(|f(u, 0,0,0)|^{2}+|g(u, 0,0,0)|^{2}+|\sigma(u, 0,0,0)|^{2}+\left|\hat{Z}_{u}^{t_{0}, x_{0}}\right|^{2}\right) d u\right|\right]<+\infty,
$$

we deduce that

$$
\lim _{(t, x) \rightarrow\left(t_{0}, x_{0}\right)} u(t, x)=u\left(t_{0}, x_{0}\right) .
$$

The continuity result of the former theorem is enough for the purpose of this paper: the link with PDEs. Nevertheless, we might like to have more regularity on the function $u$, especially in the $x$ variable. For instance, if the function $u$ is uniformly Lipschitz in $x$, we can use the same techniques as Delarue in [1] to prove existence and uniqueness of the solution of the FBSDE over $[0, T]$ for any positive $T$. 
A quick glance at the last inequality of the previous proof shows that the function $u$ is indeed Lipschitz in $x$. But, the Lipschitz constant depends on $t$ and is difficult to estimate as it involves the spectral radius of the Lipschitz matrix $A(t)$. Therefore, we will not be able to prove solvability of the FBSDE on any interval.

In [2], Delarue and Menozzi need the function $u$ to be of class $\mathcal{C}^{1,2}$ to prove convergence of their numerical scheme in order to simulate the solutions of the FBSDE. This regularity is obtained thanks to the links between FBSDEs and PDEs. If we are able to prove (by analytical techniques) uniqueness of the viscosity solution and existence of a strong solution of the PDE, then $u$ is therefore this strong solution and is consequently differentiable.

As we will next see, the links between FBSDEs and PDEs are not so precise when the coefficient $\sigma$ depends on $Z$. Nevertheless, Rivière proposed in [8] another discretization scheme that do not need so much regularity of $u$.

\section{Connection with PDEs: The general Result}

In view of the results of [6], we must consider functions $\sigma$ that do depend on $z$ in order to get a representation of solutions of PDEs with gradient dependent second order term. However, the proof of the next theorem only applies for functions $f$ and $g$ that do not depend on $z$. Consequently, let us first fix the following functions :

$$
\begin{aligned}
M & :[0,+\infty) \times \mathbb{R}^{d} \times \mathbb{R} \times \mathbb{R}^{d} \longmapsto \mathbb{R}^{d \times d}, \\
f & :[0,+\infty) \times \mathbb{R}^{d} \times \mathbb{R} \longmapsto \mathbb{R}^{d}, \\
g & :[0,+\infty) \times \mathbb{R}^{d} \times \mathbb{R} \longmapsto \mathbb{R}, \\
h & : \mathbb{R}^{d} \longmapsto \mathbb{R} .
\end{aligned}
$$

Let also fix the terminal time $T>0$. The more general quasilinear parabolic PDE we will handle is of the following type:

$$
\left\{\begin{array}{l}
\frac{\partial u}{\partial t}(t, x)+\sum_{1 \leq i, j \leq d} M(t, x, u(t, x), \nabla u(t, x))_{i j} \frac{\partial^{2} u}{\partial x_{i} \partial x_{j}}(t, x) \\
\quad+\langle f(t, x, u(t, x)), \nabla u(t, x)\rangle+g(t, x, u(t, x))=0 \quad \text { for } t \in(0, T), x \in \mathbb{R}^{d}, \\
u(T, x)=h(x) \quad \text { for } x \in \mathbb{R}^{d} .
\end{array}\right.
$$

In view of the results of [6], we surmise that, in order to get a probabilistic representation of the solutions of (5), we may consider FBSDE of the form:

$$
\left\{\begin{array}{l}
X_{s}^{t, x}=x+\int_{t}^{s} f\left(r, X_{r}^{t, x}, Y_{r}^{t, x}\right) d r+\int_{t}^{s} \sigma\left(r, X_{r}^{t, x}, Y_{r}^{t, x}, Z_{r}^{t, x}\right) d B_{r}, t \leq s \leq T, \\
Y_{s}^{t, x}=h\left(X_{T}^{t, x}\right)+\int_{s}^{T} g\left(r, X_{r}^{t, x}, Y_{r}^{t, x}\right) d r-\int_{s}^{T}\left\langle Z_{r}^{t, x}, d B_{r}\right\rangle, t \leq s \leq T .
\end{array}\right.
$$

Here, $\sigma$ is a $\mathbb{R}^{d \times d}$-valued function that we will choose later.

We suppose that the conditions of the previous section are satisfied in order to have existence and uniqueness of the solution of the system (6) and the global continuity of the function $u$. Then, we have the following result that links the PDE (5) to the FBSDE (6):

Theorem 3.1. Suppose that $\sigma$ is an invertible matrix and is related to $M$ via the following equation:

$$
\forall t, x, y, z, \quad \sigma \sigma^{*}(t, x, y, z)=2 M\left(t, x, y,\left(\sigma^{*}(t, x, y, z)\right)^{-1} z\right)
$$


We suppose moreover that the functions $f, g, h$ are globally continuous, that $M$ is globally Lipschitz and that $\sigma$ satisfies the additional assumption: there exists a positive constant $\lambda$ such that, for all $t, x, y, z, w$,

$$
\left|\sigma(t, x, y, z)^{-1} w\right| \leq \lambda|w|
$$

Let us define on $[0, T) \times \mathbb{R}^{d}$ the function $u(t, x)=Y_{t}^{t, x}$. Then $u$ is a viscosity solution of the $P D E(5)$.

Proof: The proof is an adaptation of those of [6], Theorem 5.1.

Let us denote by $\bar{B}(x, \rho)$ the closed Euclidean ball in $\mathbb{R}^{d}$ centered at $x$ and of radius $\rho$.

We must first prove the following lemma:

Lemma 3.2. We have, a.s. for every $r \geq t$,

$$
Y_{r}^{t, x}=Y_{r}^{r, X_{r}^{t, x}}=u\left(r, X_{r}^{t, x}\right) .
$$

Proof: Let us fix $t \in[0, T]$ and let $U$ be a $\mathcal{F}_{t}$-measurable random variable. We consider the FBSDE

$$
\begin{cases}X_{s}=U+\int_{t}^{s} f\left(r, X_{r}, Y_{r}, Z_{r}\right) d r+\int_{t}^{s} \sigma\left(r, X_{r}, Y_{r}, Z_{r}\right) d B_{r}, & t \leq s \leq T, \\ Y_{s}=h\left(X_{T}\right)+\int_{s}^{T} g\left(r, X_{r}, Y_{r}, Z_{r}\right) d r-\int_{s}^{T}\left\langle Z_{r}, d B_{r}\right\rangle, & t \leq s \leq T .\end{cases}
$$

It is easy to extend Theorems 2.6 and 2.7 to this case with $|x|^{2}$ replaced by $\mathbb{E}\left[|U|^{2}\right]$. We denote by $\left(X^{t, U}, Y^{t, U}, Z^{t, U}\right)$ the unique solution of (9). We claim that there exists a positive constant $C$ such that, for every $x \in \mathbb{R}^{d}$ and every $\varepsilon>0$,

$$
\mathbb{E}\left[\mathbf{1}_{|U-x|<\varepsilon}\left|Y_{t}^{t, U}-Y_{t}^{t, x}\right|^{2}\right] \leq C \mathbb{E}\left[\mathbf{1}_{|U-x|<\varepsilon}|U-x|^{2}\right] .
$$

Indeed, let us define, for $s \in[t, T]$,

$$
\bar{X}_{s}=X_{s}^{t, U}-X_{s}^{t, x}, \bar{Y}_{s}=Y_{s}^{t, U}-Y_{s}^{t, x}, \bar{Z}_{s}=Z_{s}^{t, U}-Z_{s}^{t, x} .
$$

Then, $(\bar{X}, \bar{Y}, \bar{Z})$ is solution of the FBSDE

$$
\begin{cases}\bar{X}_{s}=U-x+\int_{t}^{s} \bar{f}\left(r, \bar{X}_{r}, \bar{Y}_{r}, \bar{Z}_{r}\right) d r+\int_{t}^{s} \bar{\sigma}\left(r, \bar{X}_{r}, \bar{Y}_{r}, \bar{Z}_{r}\right) d B_{r}, & t \leq s \leq T, \\ \bar{Y}_{s}=\bar{h}\left(\bar{X}_{T}\right)+\int_{s}^{T} \bar{g}\left(r, \bar{X}_{r}, \bar{Y}_{r}, \bar{Z}_{r}\right) d r-\int_{s}^{T}\left\langle\bar{Z}_{r}, d B_{r}\right\rangle, & t \leq s \leq T\end{cases}
$$

with

$$
\begin{aligned}
\bar{f}(r, u, v, w) & =f\left(r, u+X_{r}^{t, x}, v+Y_{r}^{t, x}, w+Z_{r}^{t, x}\right)-f\left(r, X_{r}^{t, x}, Y_{r}^{t, x}, Z_{r}^{t, x}\right) \\
\bar{g}(r, u, v, w) & =g\left(r, u+X_{r}^{t, x}, v+Y_{r}^{t, x}, w+Z_{r}^{t, x}\right)-g\left(r, X_{r}^{t, x}, Y_{r}^{t, x}, Z_{r}^{t, x}\right) \\
\bar{\sigma}(r, u, v, w) & =\sigma\left(r, u+X_{r}^{t, x}, v+Y_{r}^{t, x}, w+Z_{r}^{t, x}\right)-\sigma\left(r, X_{r}^{t, x}, Y_{r}^{t, x}, Z_{r}^{t, x}\right) \\
\bar{h}(u) & =h\left(u+X_{T}^{t, x}\right)-h\left(X_{T}^{t, x}\right) .
\end{aligned}
$$

The fonctions $\bar{f}, \bar{g}, \bar{\sigma}$ and $\bar{h}$ still statisfy assumptions [H1], [H2] and [H3].

Let us consider now $A \in \mathcal{F}_{t}$ and let us set for every $r \in[t, T]$,

$$
\left(X_{r}^{A}, Y_{r}^{A}, Z_{r}^{A}\right)=\left(\mathbf{1}_{A} X_{r}, \mathbf{1}_{A} Y_{r}, \mathbf{1}_{A} Z_{r}\right) .
$$


The processes $X^{A}, Y^{A}, Z^{A}$ are still progressively measurable with respect to the filtration $\mathcal{F}$ and it is easy to see that they are solutions of the FBSDE

$$
\begin{cases}X_{s}^{A}=\mathbf{1}_{A}(U-x)+\int_{t}^{s} \mathbf{1}_{A} \bar{f}\left(r, X_{r}^{A}, Y_{r}^{A}, Z_{r}^{A}\right) d r+\int_{t}^{s} \mathbf{1}_{A} \bar{\sigma}\left(r, X_{r}^{A}, Y_{r}^{A}, Z_{r}^{A}\right) d B_{r}, & t \leq s \leq T, \\ Y_{s}^{A}=\mathbf{1}_{A} \bar{h}\left(X_{T}^{A}\right)+\int_{s}^{T} \mathbf{1}_{A} \bar{g}\left(r, X_{r}^{A}, Y_{r}^{A}, Z_{r}^{A}\right) d r-\int_{s}^{T}\left\langle Z_{r}^{A}, d B_{r}\right\rangle, & t \leq s \leq T\end{cases}
$$

We then apply Theorem 2.7 to this FBSDE which gives (after noticing that $\bar{f}(r, 0,0,0)=0$ and so for $\bar{g}, \bar{\sigma}$ and $\bar{h}$ ) that there exists a constant $C$ (that does not depend on $x$ ) such that

$$
\mathbb{E}\left[\sup _{t \leq s \leq T}\left|\mathbf{1}_{A} \bar{X}_{s}\right|^{2}\right]+\mathbb{E}\left[\left.\sup _{t \leq s \leq T}\left|\mathbf{1}_{A} \bar{Y}\right|_{s}\right|^{2}\right]+\mathbb{E}\left[\left.\int_{t}^{T} \mathbf{1}_{A} \bar{Z}_{s}\right|^{2} d s\right] \leq C \mathbb{E}\left[\mathbf{1}_{A}|U-x|^{2}\right] .
$$

In particular, we have

$$
\mathbb{E}\left[\mathbf{1}_{A}\left|Y_{t}^{t, x}-Y_{t}^{t, U}\right|^{2}\right] \leq C \mathbb{E}\left[\mathbf{1}_{A}|U-x|^{2}\right]
$$

which gives Equation (10) for $A=\{|U-x|<\varepsilon\}$.

Now, we have

$$
\begin{aligned}
& \mathbb{E}\left[\mathbf{1}_{\{|U-x|<\varepsilon\}}\left|u(t, U)-Y_{t}^{t, U}\right|^{2}\right] \leq 2 \mathbb{E}\left[\mathbf{1}_{\{|U-x|<\varepsilon\}}|u(t, U)-u(t, x)|^{2}\right] \\
&+2 \mathbb{E}\left[\mathbf{1}_{\{|U-x|<\varepsilon\}}\left|Y_{t}^{t, x}-Y_{y}^{t, U}\right|^{2}\right] \\
& \leq 2 C^{\prime} \mathbb{E}\left[\mathbf{1}_{\{|U-x|<\varepsilon\}}|U-x|^{2}\right]+2 C \mathbb{E}\left[\mathbf{1}_{\{|U-x|<\varepsilon\}}|U-x|^{2}\right]
\end{aligned}
$$

where the last inequality follows from Theorem 2.8 and inequality (10).

We end the proof by writting, for every positive integer $N$,

$$
\begin{aligned}
\mathbb{E}\left[\left|u(t, U)-Y_{t}^{t, U}\right|^{2}\right] & \leq \sum_{k \in \mathbb{Z}^{d}} \mathbb{E}\left[\mathbf{1}_{\{|U-k / N| \leq 1 / N\}}\left|u(t, U)-Y_{t}^{U}\right|^{2}\right] \\
& \leq \frac{C}{N^{2}} \sum_{k \in \mathbb{Z}^{d}} \mathbb{E}\left[\mathbf{1}_{\{|U-k / N| \leq 1 / N\}}\right] \\
& \leq \frac{C 2^{d}}{N^{2}} .
\end{aligned}
$$

As this is true for every integer $N$, we deduce that $Y_{t}^{t, U}=u(t, U)$ a.s.

We thus proved that, for every $r>t$, a.s.

$$
Y_{r}^{r, X_{r}^{t, x}}=u\left(r, X_{r}^{t, x}\right) .
$$

This equality is true a.s. for every $r>t$ by continuity of $u, X$ and $Y$. Eventually, the equality

$$
Y_{r}^{t, x}=Y_{r}^{r, X_{r}^{t, x}}
$$

is a direct consequence of uniqueness of the FBSDE.

Let us turn now to the proof of Theorem 3.1. To begin with, we will prove that $u$ is a viscosity supersolution of (5). The fact that $u$ is also a viscosity subsolution can be proved by a similar argument. So, let $\phi$ be a $\mathcal{C}^{2}$ real-valued function on $[0, T) \times \mathbb{R}^{d}$ and let $\left(t_{0}, x_{0}\right)$ be a 
local maximum of $u-\phi$. Without loss of generality, we may suppose that $(u-\phi)\left(t_{0}, x_{0}\right)=0$ and we want to prove that

$$
\begin{aligned}
\frac{\partial \phi}{\partial t}\left(t_{0}, x_{0}\right)+\sum_{1 \leq i, j \leq d} M\left(t_{0}, x_{0}, u\left(t_{0}, x_{0}\right), \nabla \phi\left(t_{0}, x_{0}\right)\right)_{i j} \frac{\partial^{2} \phi}{\partial x_{i} \partial x_{j}}\left(t_{0}, x_{0}\right) \\
+\left\langle f\left(t_{0}, x_{0}, u\left(t_{0}, x_{0}\right)\right), \nabla \phi\left(t_{0}, x_{0}\right)\right\rangle+g\left(t_{0}, x_{0}, u\left(t_{0}, x_{0}\right)\right) \geq 0 .
\end{aligned}
$$

Let us suppose that this inequality is false. In other words, by continuity, let us suppose that there exists a real number $0<\mu<T-t_{0}$ such that, for every $(t, x) \in\left[t_{0}, t_{0}+\mu\right] \times \bar{B}\left(x_{0}, \mu\right)$, we have

$$
u(t, x) \leq \phi(t, x)
$$

and

$$
\begin{aligned}
\frac{\partial \phi}{\partial t}(t, x)+\sum_{1 \leq i, j \leq d} M(t, x, u(t, x), \nabla \phi( & t, x))_{i j} \frac{\partial^{2} \phi}{\partial x_{i} \partial x_{j}}(t, x) \\
& +\langle f(t, x, u(t, x)), \nabla \phi(t, x)\rangle+g(t, x, u(t, x))<0 .
\end{aligned}
$$

Let us define the stopping time $\tau$ by:

$$
\tau=\inf \left\{s \in\left[t_{0}, t_{0}+\mu\right], X_{s}^{t_{0}, x_{0}} \notin \bar{B}\left(x_{0}, \mu\right)\right\}
$$

and let us set

$$
\left(\bar{X}_{s}, \bar{Y}_{s}, \bar{Z}_{s}\right)=\left(X_{s \wedge \tau}^{t_{0}, x_{0}}, Y_{s \wedge \tau}^{t_{0}, x_{0}}, \mathbf{1}_{\left[t_{0}, \tau\right]}(s) Z_{s}^{t_{0}, x_{0}}\right) .
$$

Let us remark that the pair $(\bar{Y}, \bar{Z})$ is solution of the BSDE

$$
\bar{Y}_{s}=u\left(\tau, X_{\tau}^{t_{0}, x_{0}}\right)+\int_{s \wedge \tau}^{\tau} g\left(r, X_{r}^{t, x}, u\left(r, X_{r}^{t, x}\right)\right) d r-\int_{s \wedge \tau}^{\tau}\left\langle\bar{Z}_{r}, d B_{r}\right\rangle .
$$

Moreover, applying Itô's formula to $\phi\left(s, X_{s}^{t_{0}, x_{0}}\right)$ leads to

$$
\begin{aligned}
\phi\left(s \wedge \tau, X_{s \wedge \tau}^{t_{0}, x_{0}}\right)=\phi\left(\tau, X_{\tau}^{t_{0}, x_{0}}\right)-\int_{s \wedge \tau}^{\tau} & \left(\frac{\partial \phi}{\partial t}\left(v, X_{v}^{t_{0}, x_{0}}\right)+L_{\sigma} \phi\left(v, X_{v}^{t_{0}, x_{0}}, u\left(v, X_{v}^{t_{0}, x_{0}}\right), Z_{v}^{t_{0}, x_{0}}\right)\right) d v \\
& -\int_{s \wedge \tau}^{\tau}\left\langle\sigma^{*}\left(v, X_{v}^{t_{0}, x_{0}}, Y_{v}^{t_{0}, x_{0}}, Z_{v}^{t_{0}, x_{0}}\right) \nabla \phi\left(v, X_{v}^{t_{0}, x_{0}}\right), d B_{v}\right\rangle
\end{aligned}
$$

where $L_{\sigma}$ denotes the differential operator

$$
L_{\sigma} \phi(s, x, y, z)=\frac{1}{2} \sum_{1 \leq i, j \leq d} \sigma \sigma^{*}(s, x, y, z)_{i j} \frac{\partial^{2} \phi}{\partial x_{i} \partial x_{j}}(s, x)+\langle f(s, x, y), \nabla \phi(s, x)\rangle .
$$

Let us set now, for $s \in\left[t_{0}, t_{0}+\mu\right]$,

$$
\left(\hat{X}_{s}, \hat{Y}_{s}, \hat{Z}_{s}\right)=\left(X_{s \wedge \tau}^{t_{0}, x_{0}}, \phi\left(s \wedge \tau, X_{s \wedge \tau}^{t_{0}, x_{0}}\right), \sigma^{*}\left(s \wedge \tau, X_{s \wedge \tau}^{t_{0}, x_{0}}, Y_{s \wedge \tau}^{t_{0}, x_{0}}, Z_{s \wedge \tau}^{t_{0}, x_{0}}\right) \nabla \phi\left(s \wedge \tau, X_{s \wedge \tau}^{t_{0}, x_{0}}\right)\right)
$$


Then, we rewrite the previous equation (in order to make the function $M$ appear) in the following form:

$$
\begin{aligned}
\hat{Y}_{s \wedge \tau}=\phi\left(\tau, X_{\tau}^{t_{0}, x_{0}}\right)- & \int_{s \wedge \tau}^{\tau}\left(\frac{\partial \phi}{\partial t}\left(v, \hat{X}_{v}\right)+L_{M} \phi\left(v, \hat{X}_{v}\right)\right) d v-\int_{s \wedge \tau}^{\tau}\left\langle\hat{Z}_{v}, d B_{v}\right\rangle \\
& -\int_{s \wedge \tau}^{\tau} \sum_{1 \leq i, j \leq d}\left[\frac{1}{2} \sigma \sigma^{*}\left(v, X_{v}^{t_{0}, x_{0}}, u\left(v, X_{v}^{t_{0}, x_{0}}\right), Z_{v}^{t_{0}, x_{0}}\right)\right. \\
& \left.\quad-M\left(v, X_{v}^{t_{0}, x_{0}}, u\left(v, X_{v}^{t_{0}, x_{0}}\right), \nabla \phi\left(v, X_{v}^{t_{0}, x_{0}}\right)\right)\right]_{i j} \frac{\partial^{2} \phi}{\partial x_{i} \partial x_{j}}\left(v, X_{v}^{t_{0}, x_{0}}\right) d v
\end{aligned}
$$

where $L_{M}$ denotes the differential operator

$$
L_{M} \phi(s, x)=\sum_{1 \leq i, j \leq d} M(s, x, u(s, x), \nabla \phi(s, x))_{i j} \frac{\partial^{2} \phi}{\partial x_{i} \partial x_{j}}(s, x)+\langle f(s, x, u(s, x)), \nabla \phi(s, x)\rangle .
$$

Now using equation (7), we have

$$
\begin{aligned}
& \frac{1}{2} \sigma \sigma^{*}\left(v, X_{v}^{t_{0}, x_{0}}, u\left(v, X_{v}^{t_{0}, x_{0}}\right), Z_{v}^{t_{0}, x_{0}}\right)= \\
& M\left(v, X_{v}^{t_{0}, x_{0}}, u\left(v, X_{v}^{t_{0}, x_{0}}\right),\left(\sigma^{*}\left(v, X_{v}^{t_{0}, x_{0}}, u\left(v, X_{v}^{t_{0}, x_{0}}\right), Z_{v}^{t_{0}, x_{0}}\right)\right)^{-1} Z_{v}^{t_{0}, x_{0}}\right)
\end{aligned}
$$

and, by the definition of $\hat{Z}$, we have

$$
\begin{aligned}
\alpha(v)= & \frac{1}{2} \sigma \sigma^{*}\left(v, X_{v}^{t_{0}, x_{0}}, u\left(v, X_{v}^{t_{0}, x_{0}}\right), Z_{v}^{t_{0}, x_{0}}\right)-M\left(v, X_{v}^{t_{0}, x_{0}}, u\left(v, X_{v}^{t_{0}, x_{0}}\right), \nabla \phi\left(v, X_{v}^{t_{0}, x_{0}}\right)\right) \\
= & M\left(v, X_{v}^{t_{0}, x_{0}}, u\left(v, X_{v}^{t_{0}, x_{0}}\right),\left(\sigma^{*}\left(v, X_{v}^{t_{0}, x_{0}}, u\left(v, X_{v}^{t_{0}, x_{0}}\right), Z_{v}^{t_{0}, x_{0}}\right)\right)^{-1} Z_{v}^{t_{0}, x_{0}}\right) \\
& \quad-M\left(v, X_{v}^{t_{0}, x_{0}}, u\left(v, X_{v}^{t_{0}, x_{0}}\right),\left(\sigma^{*}\left(v, X_{v}^{t_{0}, x_{0}}, u\left(v, X_{v}^{t_{0}, x_{0}}\right), Z_{v}^{t_{0}, x_{0}}\right)\right)^{-1} \hat{Z}_{v}\right) .
\end{aligned}
$$

Now, by the Lipschitz property of $M$ and assumption (8), we have

$$
|\alpha(v)| \leq C\left|Z_{v}^{t_{0}, x_{0}}-\hat{Z}_{v}\right|
$$

for some constant $C$. Consequently, for every index $(i, j)$, there exists a bounded $\mathbb{R}^{d}$-valued adapted process $\gamma_{u}^{i j}$ such that

$$
\alpha(v)_{i j}=\left\langle\gamma_{v}^{i j},\left(Z_{v}^{t_{0}, x_{0}}-\hat{Z}_{v}\right)\right\rangle .
$$

Eventually, let us consider the process $\bar{Y}_{s}-\hat{Y}_{s}$ which may be written as:

$$
\begin{aligned}
\bar{Y}_{s}-\hat{Y}_{s}=(u-\phi)\left(\tau, \hat{X}_{\tau}\right)+\int_{s \wedge \tau}^{\tau}( & \left.\frac{\partial \phi}{\partial t}\left(v, \hat{X}_{v}\right)+L_{M} \phi\left(v, \hat{X}_{v}\right)+g\left(v, \hat{X}_{v}, u\left(v, \hat{X}_{v}\right)\right)\right) d v \\
& +\int_{s \wedge \tau}^{\tau}\left\langle\left(\gamma \partial^{2} \phi\right)_{v}, \bar{Z}_{v}-\hat{Z}_{v}\right\rangle d v-\int_{s \wedge \tau}^{\tau}\left\langle\left(\bar{Z}_{v}-\hat{Z}_{v}\right), d B_{v}\right\rangle
\end{aligned}
$$

where $\left(\gamma \partial^{2} \phi\right)_{v}$ denotes the $\mathbb{R}^{d}$-valued process

$$
\left(\gamma \partial^{2} \phi\right)_{v}=\sum_{1 \leq i, j \leq d} \frac{\partial^{2} \phi}{\partial x_{i} \partial x_{j}}\left(v, X_{v}^{t_{0}, x_{0}}\right) \gamma_{v}^{i j}
$$


We then conclude using the ideas of [5], Theorem 1.6. We first consider the $L^{2}$ real-valued process $\beta$ defined by:

$$
\beta_{v}=\frac{\partial \phi}{\partial t}\left(v, \hat{X}_{v}\right)+L_{M} \phi\left(v, \hat{X}_{v}\right)+g\left(v, \hat{X}_{v}, u\left(v, \hat{X}_{v}\right)\right)
$$

We have then, for every $t_{0} \leq s \leq \tau$

$$
\begin{aligned}
\bar{Y}_{s}-\hat{Y}_{s} & =(u-\phi)\left(\tau, \hat{X}_{\tau}\right)+\int_{s \wedge \tau}^{\tau}\left(\beta_{v}+\left\langle\left(\gamma \partial^{2} \phi\right)_{v}, \bar{Z}_{v}-\hat{Z}_{v}\right\rangle\right) d v-\int_{s \wedge \tau}^{\tau}\left\langle\left(\bar{Z}_{v}-\hat{Z}_{v}\right), d B_{v}\right\rangle \\
& =\mathbb{E}\left[\Gamma_{s, \tau}\left(\bar{Y}_{\tau}-\hat{Y}_{\tau}\right)+\int_{s}^{\tau} \Gamma_{s, v} \beta_{v} d v \mid \mathcal{F}_{s}\right]
\end{aligned}
$$

where

$$
\Gamma_{s, v}=\exp \left(\int_{s}^{v}\left\langle\left(\gamma \partial^{2} \phi\right)_{u}, d B_{u}\right\rangle-\frac{1}{2} \int_{s}^{v}\left|\left(\gamma \partial^{2} \phi\right)_{u}\right|^{2} d u\right) .
$$

In particular, we get

$$
\bar{Y}_{t_{0}}-\hat{Y}_{t_{0}}=(u-\phi)\left(t_{0}, x_{0}\right)=\mathbb{E}\left[\Gamma_{t_{0}, \tau}\left(\bar{Y}_{\tau}-\hat{Y}_{\tau}\right)+\int_{t_{0}}^{\tau} \Gamma_{t_{0}, v} \beta_{v} d v\right] .
$$

To conclude, we must remark that, by the definition of $\tau$ we have

$$
\bar{Y}_{\tau}-\hat{Y}_{\tau} \leq 0 \quad \text { and } \quad \beta_{v}<0 \text { for every } t_{0} \leq v<\tau .
$$

Consequently, we have that $(u-\phi)\left(t_{0}, x_{0}\right)<0$ which contradicts our first assumption.

The problem now is to find some conditions on $M$ such that there exists a function $\sigma$ that satisfies the condition (7). An obvious necessary condition is that, for every $(t, x, y, z)$, $M(t, x, y, z)$ must be a symmetric invertible matrix. But a sufficient condition is much more involved and we will restrict ourselves to very specific cases.

4. The one dimensional Case: Case of an increasing function $M$ on $[0,+\infty)$.

We will take here $d=1$ and consequently, all the considered functions will be real valued. Moreover, as equation (7) only involves the $z$-dependence of $M$ and does not depend on $f$ and $g$, we may restrict our attention to the following one-dimensional PDE:

$$
\left\{\begin{array}{l}
\frac{\partial u}{\partial t}(t, x)+M\left(\frac{\partial u}{\partial x}(t, x)\right) \frac{\partial^{2} u}{\partial x^{2}}(t, x)=0, \quad 0<t<T, \quad x \in \mathbb{R}, \\
u(T, x)=h(x), \quad x \in \mathbb{R},
\end{array}\right.
$$

where $M$ and $h$ are real-valued functions defined respectively on $[0, T) \times \mathbb{R}$ and $\mathbb{R}$.

The condition (7) now consists in finding a function $\sigma: \mathbb{R} \longrightarrow \mathbb{R}$ such that, for every $x \in \mathbb{R}$, we have

$$
\sigma(x)^{2}=2 M\left(\frac{x}{\sigma(x)}\right) .
$$

Moreover, this function $\sigma$ must be chosen such that the next system of FBSDE has a unique solution. This system is:

$$
\left\{\begin{array}{l}
X_{s}^{t, x}=x+\int_{t}^{s} \sigma\left(Z_{r}^{t, x}\right) d B_{r} \quad t \leq s \leq T \\
Y_{s}^{t, x}=h\left(X_{T}^{t, x}\right)-\int_{s}^{T} Z_{r}^{t, x} d B_{r} \quad t \leq s \leq T
\end{array}\right.
$$


An adaptation of the proof of [6], Theorem 3.1 leads to the following condition:

Proposition 4.1. If $\sigma$ and $h$ are Lipschitz functions with Lipschitz coefficient $k_{\sigma}$ and $k_{h}$ respectively, and if $k_{\sigma} k_{h}<1$, then the FBSDE (13) has a unique solution.

We will only consider functions $M$ which satisfy the next conditions:

(A1) $M$ is an even function,

(A2) $M$ is strictly increasing on $[0,+\infty)$,

(A3) $M(0)>0$

(A4) $M$ is continuously differentiable on $(0,+\infty)$,

(A5) the zeros of $M^{\prime}$ are isolated,

(A6) $M^{\prime}$ has a right limit at 0.

In the sequel, we adopt the convention $M(\infty)=\lim _{t \rightarrow+\infty} M(t) \in \mathbb{R} \cup\{+\infty\}$.

Proposition 4.2. Let us suppose that $M$ satisfies (A1)-(A6). Then the function $\Psi$ defined by:

$$
\Psi:\left\{\begin{array}{l}
{[\sqrt{2 M(0)} ; \sqrt{2 M(\infty)}) \rightarrow[0,+\infty)} \\
x \rightarrow x M^{-1}\left(\frac{x^{2}}{2}\right)
\end{array}\right.
$$

is a strictly increasing one-to-one function.

Moreover, $\Psi^{-1}$ is Lipschitz and its Lipschitz constant is $\frac{1}{\alpha}$

where $\alpha=\inf _{t>0}\left\{t+2 \frac{M(t)}{M^{\prime}(t)}\right\}>0$.

Proof: We obtain that $\Psi$ is increasing as a composition and product of positive increasing functions. Moreover, it is obvious that $\Psi$ maps its domain onto $[0,+\infty)$.

By deriving $\Psi^{-1}$, we obtain that, out of some isolated points of $[\sqrt{2 M(0)} ; \sqrt{2 M(\infty)})$,

$$
\left[\Psi^{-1}\right]^{\prime}(\Psi(t))=\frac{1}{M^{-1}\left(t^{2} / 2\right)+t^{2} / M^{\prime}\left(M^{-1}\left(t^{2} / 2\right)\right)} .
$$

Considering $t=\sqrt{2 M(u)}$ with $u \in[0,+\infty)$, we obtain that $\Psi$ is a Lipschitz function if and only if $\inf _{t>0}\left\{t+2 \frac{M(t)}{M^{\prime}(t)}\right\}>0$ that is to say if and only if $\liminf _{t \rightarrow 0+} \frac{M(t)}{M^{\prime}(t)}>0$, which is the case by assumptions (A2), (A3) and (A6).

Let us define the even function $\sigma$ which is equal to $\Psi^{-1}$ on $[0,+\infty)$.

The function $\sigma$ is a Lipschitz function whose Lipschitz constant is $\frac{1}{\alpha}$, which maps $\mathbb{R}$ onto the interval $[\sqrt{2 M(0)}, \sqrt{2 M(\infty)})$.

Moreover, for every $x \in \mathbb{R}$ we have $\frac{1}{2} \sigma(x)^{2}=M\left(\frac{x}{\sigma(x)}\right)$ and, by assumption (A3), the condition (8) is satisfied.

Consequently, we have the following result:

Proposition 4.3. Let us suppose that $M$ satisfies the assumptions $(A 1)-(A 6)$ and that $h$ is a Lipschitz function with Lipschitz constant less than $\alpha$. Let $\sigma$ be defined as previously. Then, the FBSDE (13) has a unique solution and the function $u(t, x)=Y_{t}^{t, x}$ is a viscosity solution of the PDE (11) for T small enough. 


\section{Some Partial RESUlts in two Dimensions}

As announced before, the problem in higher dimension is much more difficult. We will only consider two very specific cases where the methods used in dimension one can be adapted. In both cases, we still consider a simplified equation where $M$ depends only on the gradient of $u$ and the function $f$ and $g$ are not present. Moreover, we suppose that the matrix $M$ is diagonal. If we write

$$
M(x, y)=\left(\begin{array}{cc}
\xi_{1}(x, y) & 0 \\
0 & \xi_{2}(x, y)
\end{array}\right)
$$

then the associated PDE is now

$$
\left\{\begin{array}{l}
\frac{\partial u}{\partial t}(t, x, y)+\xi_{1}(\nabla u(t, x, y)) \frac{\partial^{2} u}{\partial x^{2}}+\xi_{2}(\nabla u(t, x, y)) \frac{\partial^{2} u}{\partial y^{2}} \quad \text { for } t \in(0, T),(x, y) \in \mathbb{R}^{2} \\
u(T, x, y)=h(x, y) \quad \text { for }(x, y) \in \mathbb{R}^{2} .
\end{array}\right.
$$

In that case, the matrix $\sigma$ that satisfies equation (7) is also diagonal by the Cholesky decomposition of $M$.

We will here only focus on finding a function $\sigma$ that satisfies equation (7) and will not deal with its regularity.

5.1. Case 1: One entry of $M$ is constant. We will focus here on the case where $M$ is diagonal and one entry of $M$ is constant equal to $\varepsilon>0$ (we cannot take $\varepsilon=0$ and consider a degenerate PDE as the matrix $\sigma$ must be invertible).

Let us consider a function $\psi: \mathbb{R}^{2} \longrightarrow \mathbb{R}$ and let us study here the PDE

$$
\frac{\partial u}{\partial t}+\psi(\nabla u) \frac{\partial^{2} u}{\partial x^{2}}+\varepsilon \frac{\partial^{2} u}{\partial y^{2}}=0 .
$$

We have then

$$
M(x, y)=\left(\begin{array}{cc}
\psi(x, y) & 0 \\
0 & \varepsilon
\end{array}\right)
$$

and we have to find $\sigma$ diagonal such that

$$
\sigma(x, y)^{2}=2 M\left((\sigma(x, y))^{-1} \cdot(x, y)\right) .
$$

It is quite obvious that, if for every $y \in \mathbb{R}$, the function $x \longmapsto \psi\left(x, \frac{y}{\sqrt{2 \varepsilon}}\right)$ satisfies the assumptions of Proposition 4.2, then there exists a function $\sigma_{1}$ such that

$$
\sigma_{1}(x, y)^{2}=2 \psi\left(\frac{x}{\sigma_{1}(x, y)}, \frac{y}{\sqrt{2 \varepsilon}}\right)
$$

and the matrix $\sigma$ defined by

$$
\sigma(x, y)=\left(\begin{array}{cc}
\sigma_{1}(x, y) & 0 \\
0 & \sqrt{2 \varepsilon}
\end{array}\right)
$$

satisfies equation (7).

5.2. Case 2: The function $M$ is invertible. Let us make the following assumptions on the function $\xi(x, y)=\left(\xi_{1}(x, y), \xi_{2}(x, y)\right)$ :

[A1] The function $\xi$ is invariant under $(x, y) \mapsto(-x, y)$ and $(x, y) \mapsto(x,-y)$.

[A2] For every $(x, y) \in(0,+\infty)^{2}, \xi_{1}(x, y)=x+\alpha_{1}(x, y)$ and $\xi_{2}(x, y)=y+\alpha_{2}(x, y)$ where $\alpha_{1}$ and $\alpha_{2}$ are nonnegative functions of class $\mathcal{C}^{1}$. 
[A3] The derivatives of $\alpha_{1}$ and $\alpha_{2}$ satisfy

$$
0 \leq \frac{\partial \alpha_{1}}{\partial x}<\frac{1}{2} \quad 0 \leq \frac{\partial \alpha_{2}}{\partial y}<\frac{1}{2}, \quad\left|\frac{\partial \alpha_{1}}{\partial y}\right|<\frac{1}{2}, \quad\left|\frac{\partial \alpha_{2}}{\partial x}\right|<\frac{1}{2} .
$$

[A4] $\alpha_{1}(0,0)>0$ and $\alpha_{2}(0,0)>0$.

Under these assumptions, the function $\alpha(x, y)=\left(\alpha_{1}(x, y), \alpha_{2}(x, y)\right)$ is Lipschitz with Lipschitz constant less that 1 . Consequently, a well-known result gives that the function $\xi$ is invertible on $[0,+\infty)^{2}$. Let us denote by $\beta=\left(\beta_{1}, \beta_{2}\right)$ its inverse function, defined on some domain $V$ of $(0,+\infty)^{2}$. Moreover the Jacobian of $\xi$ is always positive, consequently, $\beta$ is differentiable on $V$ and its differential application is the inverse of the differential application of $\xi$. In particular, if we denote by $|D \xi|$ the Jacobian of $\xi$, we have

$$
\frac{\partial \beta_{1}}{\partial x}=\frac{1}{|D \xi \circ \beta|}\left(1+\frac{\partial \alpha_{2}}{\partial y} \circ \beta\right) \quad \text { and } \quad \frac{\partial \beta_{2}}{\partial y}=\frac{1}{|D \xi \circ \beta|}\left(1+\frac{\partial \alpha_{1}}{\partial x} \circ \beta\right) .
$$

As in the one-dimensional case, let us denote by $\Psi$ the function

$$
\Psi:\left\{\begin{array}{l}
V \longrightarrow[0,+\infty)^{2} \\
(x, y) \longmapsto\left(x \beta_{1}\left(x^{2} / 2, y^{2} / 2\right), y \beta_{2}\left(x^{2} / 2, y^{2} / 2\right)\right) .
\end{array}\right.
$$

The Jacobian matrix of $\Psi$ is

$$
D \psi(x, y)=\left(\begin{array}{cc}
\beta_{1}\left(x^{2} / 2, y^{2} / 2\right)+x^{2} \frac{\partial \beta_{1}}{\partial x}\left(x^{2} / 2, y^{2} / 2\right) & x y \frac{\partial \beta_{1}}{\partial y}\left(x^{2} / 2, y^{2} / 2\right) \\
x y \frac{\partial \beta_{2}}{\partial x}\left(x^{2} / 2, y^{2} / 2\right) & \beta_{2}\left(x^{2} / 2, y^{2} / 2\right)+y^{2} \frac{\partial \beta_{2}}{\partial y}\left(x^{2} / 2, y^{2} / 2\right)
\end{array}\right)
$$

and its Jacobian is (all the functions are evaluated at $\left(x^{2} / 2, y^{2} / 2\right)$ )

$$
|D \psi(x, y)|=\beta_{1} \beta_{2}+\left(x^{2} \beta_{2} \frac{\partial \beta_{1}}{\partial x}+y^{2} \beta_{1} \frac{\partial \beta_{2}}{\partial y}\right)+x^{2} y^{2}\left(\frac{\partial \beta_{1}}{\partial x} \frac{\partial \beta_{2}}{\partial y}-\frac{\partial \beta_{1}}{\partial y} \frac{\partial \beta_{2}}{\partial x}\right) .
$$

By definition, we have $\beta_{1} \beta_{2} \geq 0$. By the expression of $\frac{\partial \beta_{1}}{\partial x}$ and $\frac{\partial \beta_{2}}{\partial y}$ and assumption [A2], all the terms in the second term of $|D \psi|$ are nonnegative. Eventually, the third term is equal to

$$
x^{2} y^{2}|D \beta|=\frac{x^{2} y^{2}}{|D \xi|}
$$

which is positive since $(0,0) \notin V$ by assumption [A4].

Consequently, the Jacobian of the function $\Psi$ is always positive and the function $\Psi$ is everywhere locally invertible. By the Borel-Lebesgue characterization of compact sets, we may then define a function $\lambda(x, y)=\left(\lambda_{1}(x, y), \lambda_{2}(x, y)\right)$ on the image of $\Psi$, which is locally invertible everywhere and whose inverse is $\Psi$.

If moreover the image of $\Psi$ is $[0,+\infty)^{2}$, it is easy to see that the matrix $\sigma$ defined by

$$
\sigma(x, y)=\left(\begin{array}{cc}
\lambda_{1}(x, y) & 0 \\
0 & \lambda_{2}(x, y)
\end{array}\right)
$$

on $[0,+\infty)^{2}$ and extended to $\mathbb{R}^{2}$ by symmetry, satisfies equation $(7)$.

\section{AnOther Representation}

As the last two sections illustrate, condition (7) is rather difficult to handle and highly restrictive. In view of Ma, Protter and Yong's work [3], we can try to use another type of 
FBSDE to represent solutions of equation (5). Indeed, Ma, Protter and Yong studied the relationship between solutions of the FBSDE

$$
\left\{\begin{array}{l}
\widetilde{X}_{s}^{t, x}=x+\int_{t}^{s} f\left(r, \widetilde{X}_{r}^{t, x}, \widetilde{Y}_{r}^{t, x}\right) d r+\int_{t}^{s} \sigma\left(r, \widetilde{X}_{r}^{t, x}, \widetilde{Y}_{r}^{t, x}, \widetilde{Z}_{r}^{t, x}\right) d B_{r}, t \leq s \leq T \\
\tilde{Y}_{s}^{t, x}=h\left(\widetilde{X}_{T}^{t, x}\right)+\int_{s}^{T} g\left(r, \widetilde{X}_{r}^{t, x}, \widetilde{Y}_{r}^{t, x}\right) d r-\int_{s}^{T}\left\langle\widetilde{Z}_{r}^{t, x}, \sigma\left(r, \widetilde{X}_{r}^{t, x}, \widetilde{Y}_{r}^{t, x}, \widetilde{Z}_{r}^{t, x}\right) d B_{r}\right\rangle,
\end{array}\right.
$$

and strong solutions of the PDE

$$
\left\{\begin{array}{l}
\frac{\partial u}{\partial t}(t, x)+\sum_{1 \leq i, j \leq d} \sigma \sigma^{*}(t, x, u(t, x), \nabla u(t, x))_{i j} \frac{\partial^{2} u}{\partial x_{i} \partial x_{j}}(t, x) \\
\quad+\langle f(t, x, u(t, x)), \nabla u(t, x)\rangle+g(t, x, u(t, x))=0 \quad \text { for } t \in(0, T), x \in \mathbb{R}^{d}, \\
u(T, x)=h(x) \quad \text { for } x \in \mathbb{R}^{d},
\end{array}\right.
$$

when the coefficients $f, g, \sigma$ are smooth and $\sigma$ is non-degenerate. We see that condition (7) has disappeared with this representation.

Let us see how the method used in the present paper can be adapted to this kind of FBSDE with Lipschitz coefficients. We will only focus on the one-dimensional case $(d=1)$ in order to simplify the notations.

To begin with, for the waveform relaxation method, we must consider a new function $\widetilde{\psi}$ defined by

$$
(\widetilde{X}, \widetilde{Y}, \widetilde{Z})=\widetilde{\psi}(U, V, W)
$$

where

$$
\left\{\begin{array}{l}
\widetilde{X}_{t}=x+\int_{0}^{t} f\left(s, U_{s}, V_{s}, W_{s}\right) d s+\int_{0}^{t} \sigma\left(s, U_{s}, V_{s}, W_{s}\right) d B_{s} \\
\widetilde{Y}_{t}=h\left(\widetilde{X}_{T}\right)+\int_{t}^{T} g\left(s, U_{s}, V_{s}, W_{s}\right) d s-\int_{t}^{T} \widetilde{Z}_{s} \sigma\left(s, U_{s}, V_{s}, W_{s}\right) d B_{s} .
\end{array}\right.
$$

We must note that, for $\widetilde{Z}$ to be well-defined in the last expression, we must suppose that $\sigma$ is non-degenerate. We still suppose that $f, g, h$ and $\sigma$ satisfy assumptions $[H 1],[H 2]$ and [H3]. Then, the estimates of $\|\Delta \widetilde{X}\|_{c}$ and $\|\Delta \widetilde{Y}\|_{c}$ are exactly the same as in the proof of Proposition 2.5. But, the coefficient $\sigma$ in the stochastic integral that defines $\widetilde{Z}$ prevents us from using the same method to estimate $\|\Delta \widetilde{Z}\|_{2}^{2}$. Consequently, we are not able to prove existence and uniqueness of solutions of the FBSDE (15) nor the continuity of the function $\widetilde{u}(t, x):=\widetilde{Y}_{t}^{t, x}$.

However, the proof of Theorem 3.1 can easily be adapted to get the following theorem:

Theorem 6.1. Suppose that $\sigma$ is non-degenerate, that the coefficients $f, g, \sigma, h$ are globally continuous and that $\sigma$ satisfies (8), that there exists a unique solution to the FBSDE (15) and that the function $\widetilde{u}$ is continuous. Then $\widetilde{u}$ is a viscosity solution of the PDE (16).

\section{REFERENCES}

[1] F. Delarue. On the existence and uniqueness of solutions to fbsdes in a non-degenerate case. Stochastic Process. Appl., 99:209-286, 2002. 
[2] F. Delarue and S. Menozzi. A forward-backward algorithm for quasi-linear pde. Annals Applied Probab., To appear, 2005.

[3] J. Ma, P. Protter, and J. Yong. Solving forward-backward stochastic differential equations explicitely - a four step scheme. Probab. Th. Rel. Fields, 98:339-359, 1994.

[4] J. Ma and J. Yong. Forward-backward stochastic differential equations and their applications. In Lecture Notes Math., volume 1702. Springer, Berlin, 1999.

[5] E. Pardoux. Backward stochastic differential equations and viscosity solutions of systems of semilinear parabolic and elliptic pdes of second order. In Stochastic Analysis and Relates Topics: The Geilo Workshop, pages 79-127. Birkhäuser, 1996.

[6] E. Pardoux and S. Tang. Forward-backward stochastic differential equations and quasilinear parabolic pdes. Probab. Th. Rel. Fields, 114:123-150, 1999.

[7] P. Perona and J. Malik. Scale space and edge detection using anisotropic diffusion. IEEE Trans. Pattern Anal. Machine Intell., 12:629-639, 1990.

[8] O. Rivière. Equations différentielles stochastiques progressives rétrogrades : équations aux dérivées partielles et discrétisation. PhD thesis, Université René Descartes - Paris 5, 2005.

UFR de Mathématiques et D’Informatique, Université René Descartes, 45 rue des Saints PÈres, 75270 Paris Cedex 06, France 